\title{
Recurrent Acute Kidney Injury with Severe Loin Pain and Patchy Renal Ischaemia after Anaerobic Exercise without Renal Hypouricaemia in a New Zealand European Male
}

\author{
Richard Kelly ${ }^{a}$ David Semple ${ }^{b, c}$ Alana Harper ${ }^{d, e}$ \\ ${ }^{a}$ Auckland District Health Board, Auckland, New Zealand; 'b Department of Renal Medicine, \\ Auckland District Health Board, Auckland, New Zealand; 'School of Medicine, University of \\ Auckland, Auckland, New Zealand; dAdult Emergency Department, Auckland District Health \\ Board, Auckland, New Zealand; eAuckland Rescue Helicopter Trust, Auckland, New Zealand
}

\section{Keywords}

Acute kidney injury - Loin pain · Patchy renal ischaemia - Anaerobic exercise - Without renal hypouricaemia

\section{Abstract}

Acute kidney injury with severe loin pain and patchy renal ischaemia after anaerobic exercise (ALPE) is a rare clinical syndrome. ALPE has predominantly been described in Japanese and Korean populations to date. Many cases and most recurrent examples are associated with renal hypouricaemia. We describe a 28 -year-old New Zealand European male without renal hypouricaemia who developed recurrent ALPE whilst performing elite-level sport. Avoiding elite-level anaerobic exercise was successful at preventing further episodes. This report confirms the first known case of ALPE in a New Zealand European male and raises the possibility that ALPE is an under-recognized condition. Long-term outcomes of recurrent ALPE remain unclear, and preventative strategies should be implemented to preserve renal function. Avoiding intense anaerobic exercise is an effective preventative strategy.

\section{Introduction}

The first case of acute kidney injury (AKI) with loin pain after anaerobic exercise was described in 1981 [1]. After that, reports of similar cases began to accumulate. In the most extensive review to date, Ishikawa reviewed 118 cases and described the following features 
of ALPE [1]: (1) the patients are mostly young males; (2) renal hypouricaemia is a predisposing factor; (3) severe loin or abdominal pain develops several hours after intense anaerobic exercise; (4) laboratory tests show AKI without significant rhabdomyolysis; (5) delayed computed tomography (CT) after administration of contrast medium shows patchy wedgeshaped enhancements; and (6) the short-term prognosis is good.

This report describes recurrent ALPE without renal hypouricaemia in a New Zealand European male. The diagnosis was difficult to make because of the lack of knowledge of ALPE in New Zealand and would have been missed had the patient not presented for a second time.

\section{Case Report}

A 28-year-old New Zealand European male was admitted to our hospital with two episodes of AKI whilst participating in selection testing for an elite-level sporting team. He was normally fit and well with no family history of renal disease. His premorbid renal function was normal, with a serum creatinine of $87 \mu \mathrm{mol} / \mathrm{L}$ and an estimated glomerular filtration rate $>90 \mathrm{~mL} / \mathrm{min} / 1.73 \mathrm{~m}^{2}$. His premorbid serum uric acid was $0.38 \mathrm{mmol} / \mathrm{L}$. He had not previously engaged in elite-level training or competition.

After performing an intense anaerobic upper body exercise, the patient developed bilateral loin pain, nausea, and vomiting. He ingested $400 \mathrm{mg}$ of ibuprofen. The symptoms persisted beyond 24 hours, at which point he presented to the emergency department. There was no history of preceding flu-like symptoms. He had no myalgia, and his urine was of normal colour and volume. Physical examination was unremarkable; he was normotensive, and no costovertebral tenderness was appreciated. Laboratory tests revealed an AKI with an elevated serum creatinine level of $509 \mu \mathrm{mol} / \mathrm{L}$. Creatine kinase and uric acid were mildly elevated at $369 \mathrm{IU} / \mathrm{L}$ and $0.49 \mathrm{mmol} / \mathrm{L}$, respectively. Complete blood count was normal. Urinalysis was positive for microscopic haematuria and negative for myoglobinuria and proteinuria. Ultrasound of the renal tract was unremarkable except for mild renal echogenicity. The diagnosis was thought to be a multifactorial AKI due to dehydration, nonsteroidal anti-inflammatory drugs (NSAIDs), and a mild degree of rhabdomyolysis. The patient's symptoms and renal function improved with intravenous fluids, paracetamol, and anti-emetics. He was discharged after 48 hours and advised to keep well hydrated, avoid NSAIDs, and avoid strenuous exercise until his renal function normalized. The creatinine level returned to normal limits, defined as $<110 \mu \mathrm{mol} / \mathrm{L}$, after three weeks. However, it fluctuated between 100 and $130 \mu \mathrm{mol} / \mathrm{L}$ when he recommenced his training without any symptoms (Fig. 1).

Three months later, the patient suffered from a second episode of bilateral loin pain, nausea, and vomiting after the same intense anaerobic exercise. He did not ingest any NSAIDs this time and presented to the hospital within a couple of hours of symptom onset. $\mathrm{He}$ was mildly hypovolaemic on examination with a blood pressure of 91/56 mm $\mathrm{Hg}$ and a heart rate of 110 beats per minute. The laboratory findings on admission are shown in Table 1. An AKI was evident with a serum creatinine of $182 \mu \mathrm{mol} / \mathrm{L}$. Creatine kinase and uric acid were mildly elevated at $250 \mathrm{IU} / \mathrm{L}$ and $1.32 \mathrm{mmol} / \mathrm{L}$, respectively. There was a reactive leucocytosis. Inflammatory markers were normal. Streptococcal serology was negative. Urinalysis was positive for microscopic haematuria and proteinuria with a protein/creatinine ratio of $110 \mathrm{mg} / \mu \mathrm{mol}$ and negative for myoglobinuria. A dual-phase contrast-enhanced CT scan of the kidneys was performed, revealing patchy areas of hypoperfusion (Fig. 2).

After reviewing the medical literature, ALPE was diagnosed based on the characteristic history, laboratory findings, and CT findings. Both AKI episodes were due to this

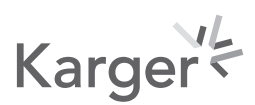




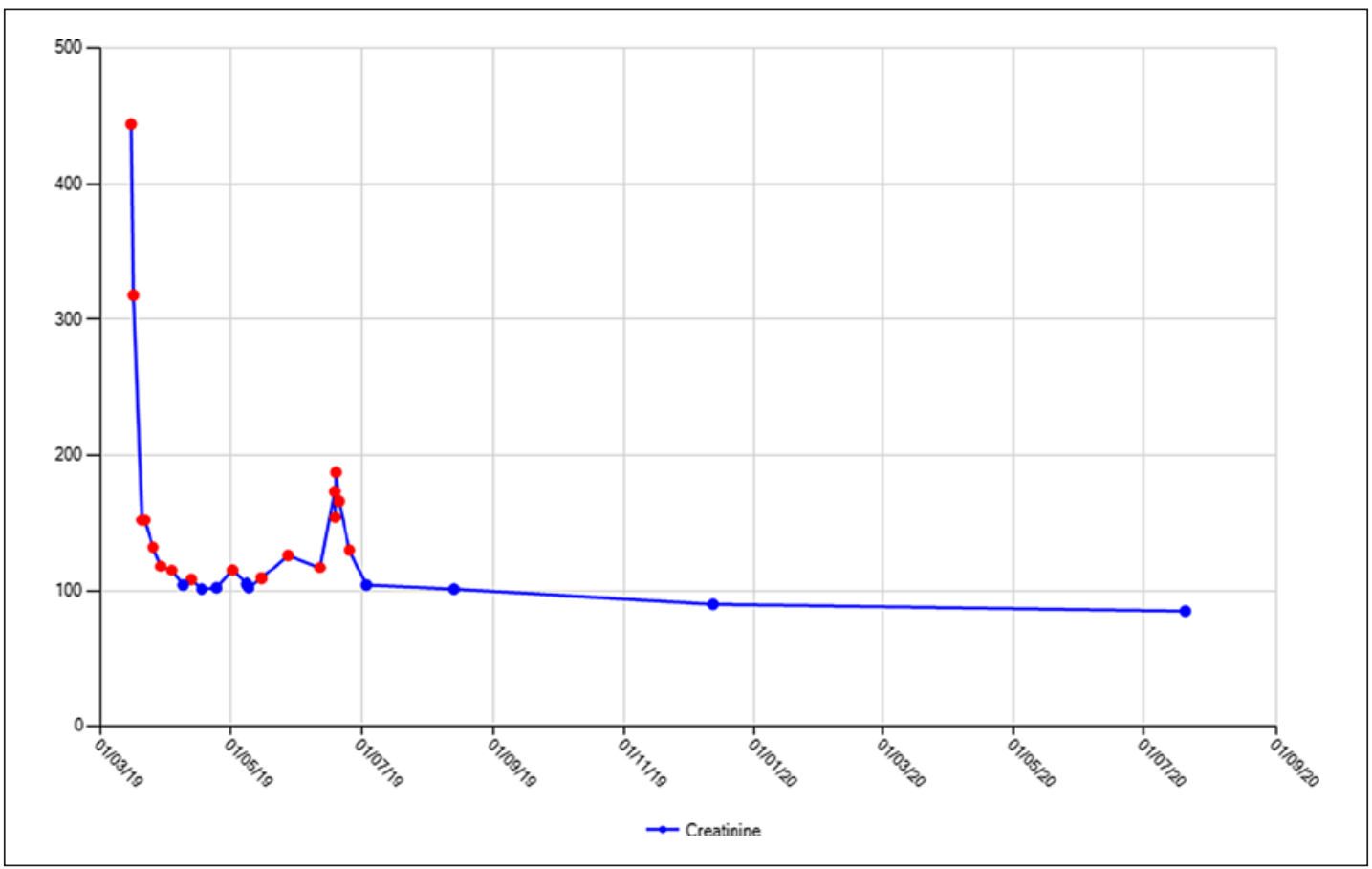

Fig. 1. Trend in serum creatinine $(\mu \mathrm{mol} / \mathrm{L})$ over time.

condition with variable levels of concomitant dehydration. The patient was discharged after his renal function improved with intravenous fluids. The creatinine level returned to normal limits after two weeks. Ongoing management and care included input from both renal and sports medicine specialists. In order to prevent ALPE recurrence, the patient was advised to avoid elite-level anaerobic exercise. He subsequently ended his elite-level sports career, which was very disappointing. However, he was able to undertake normal and even relatively high-level exercise without symptoms or alteration in his renal function when followed up at one month, six months, and one year post-discharge (Fig. 1). The serum uric acid level was $0.25 \mathrm{mmol} / \mathrm{L}$ at the one-year follow-up with a corresponding serum creatinine of $85 \mu \mathrm{mol} / \mathrm{L}$, again confirming that he did not have renal hypouricaemia.

\section{Discussion}

ALPE has predominantly been described in Japanese and Korean populations to date. It has been suggested that this trend is due to a higher prevalence of renal hypouricaemia in Japan and Korea [1-3]. However, international data on the prevalence of renal hypouricaemia is lacking, and half of ALPE cases do not have renal hypouricaemia [1]. Alternatively, this trend may reflect an increased awareness and recognition of ALPE in these countries. A few isolated cases of ALPE have been described in European [4-6] and Middle Eastern populations $[7,8]$. This report confirms the first known case of ALPE in a New Zealand European male. We suspect that ALPE is possibly under-recognized in populations around the world and may not be ascribable to specific population genotypes.

Patients with ALPE present with severe loin or abdominal pain that develops several hours after anaerobic exercise, typically track sprinting [1-3]. The pain is sharp and stabbing 
Case Reports

in Nephrology and Dialysis

సैন

+ + + + a

को

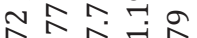

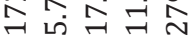

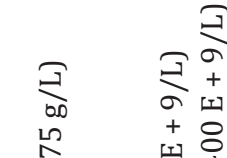

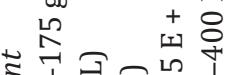

ริ

$\tau=r+\sigma]$

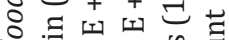

응

ปั

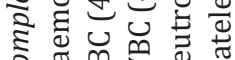

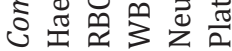

Case Rep Nephrol Dial 2021;11:176-182

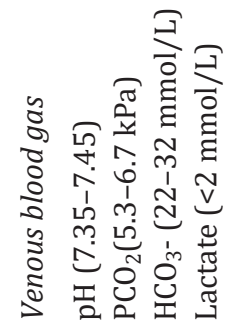

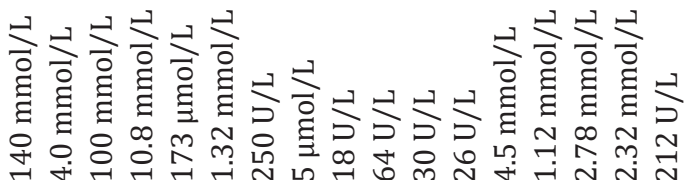

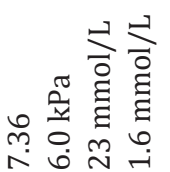

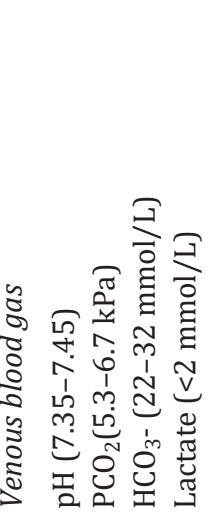

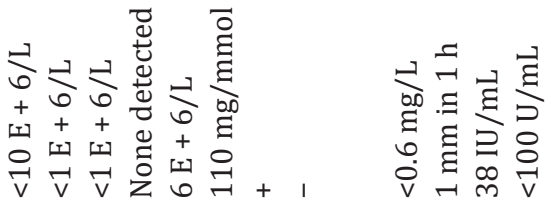

코

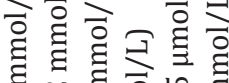

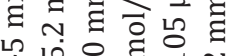

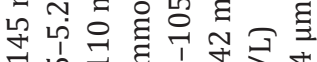

ㄴำ

0
0

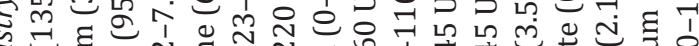

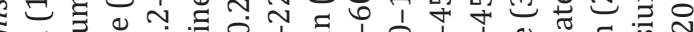

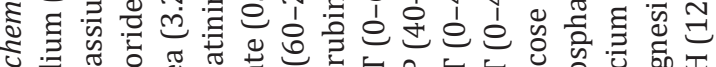

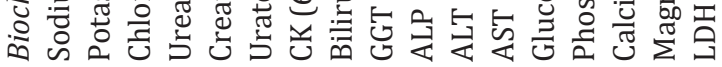
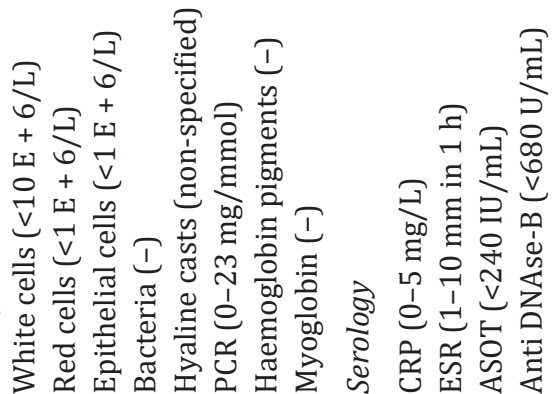
Fig. 2. CT performed immediately after contrast administration revealing patchy renal hypoperfusion (red arrows). CT, computed tomography.

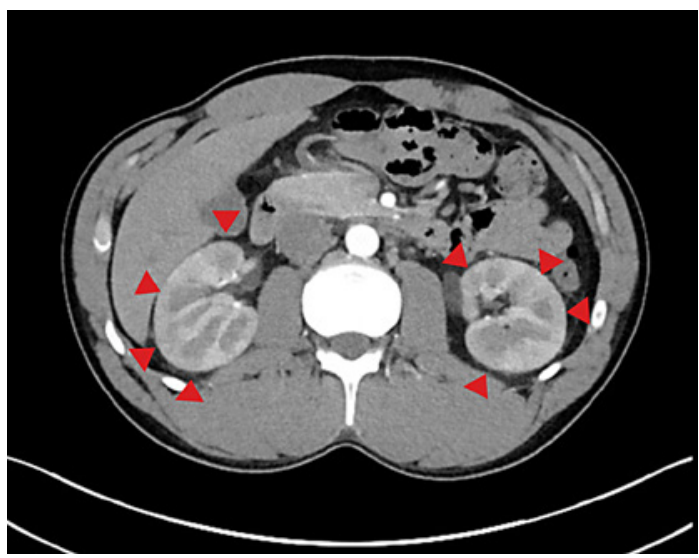

in nature and lasts one to five days [1]. Associated symptoms can include nausea and vomiting, a low-grade fever, fatigue, and prodromal flu-like symptoms [1-3]. Urine volume and colour are normal or in keeping with mild dehydration [1]. Myoglobin-induced AKI due to exertional rhabdomyolysis is an important differential diagnosis. In comparison, this condition occurs after intense aerobic exercise, typically marathon running, and patients present with myalgia, low urine output, and reddish-brown urine [9].

Laboratory tests in ALPE show AKI without significant rhabdomyolysis. Creatinine levels are elevated, whilst creatine kinase and myoglobin levels are normal or only slightly elevated (no greater than nine and seven times the upper limit of normal, respectively) [1]. Approximately half of ALPE cases are associated with renal hypouricaemia [1]. The present case did not have renal hypouricaemia, with a normal serum uric acid level measured premorbid and after the creatinine level returned to normal. Approximately one quarter of cases have abnormal urinalysis results consistent with acute tubular necrosis [1], and most renal biopsy samples also show acute tubular necrosis $[1,3]$.

A delayed renal CT scan taken 24-72 hours after contrast administration is recommended to confirm patchy wedge-shaped enhancements in the kidneys [1-3]. This finding is relatively specific for ALPE, reflecting patchy renal ischaemia [1-3]. Contrast does not initially enter the ischaemic areas because of hypoperfusion. Following reperfusion, contrast can enter the ischaemic areas, but then excretion is delayed due to renal function impairment. A CT scan taken immediately after contrast administration may capture the initial hypoperfusion phase, as in the present case, but this is variable [2]. Alternative imaging methods should be considered in patients with severe renal dysfunction to avoid contrast use. Colour Doppler ultrasound [10], bone scintigraphy with 99mTc-methylene disphosphonate [11], and diffusion-weighted magnetic resonance imaging [12] all perform well at detecting patchy renal ischaemia.

The pathogenesis of ALPE is not entirely understood. Anaerobic exercise appears to induce vasoconstriction of intra-renal arteries, leading to patchy renal ischaemia, acute tubular necrosis, and loin pain [1-3]. Although the mechanism of vasoconstriction is unclear, two hypotheses have been proposed. The first hypothesis is that type two muscle fibres are damaged by anaerobic exercise and release non-myoglobin vasoactive mediators [1]. The second hypothesis is that oxygen free radicals, which have a vasoactive function, accumulate with anaerobic exercise [1-3]. Plasma uric acid is a powerful antioxidant [13], and therefore, renal hypouricaemia may enhance the latter mechanism by reducing oxygen free radical elimination. Concurrent NSAID use and hypovolaemia may worsen the ischaemic injury by 
decreasing renal perfusion [1]. Uric acid nephropathy due to exertional changes in uric acid excretion was previously considered to have a pathogenic role [1-3]. However, no patients demonstrated uric acid crystallisation on renal biopsy in the reviews by Ishikawa and Ohta et al. [1, 3].

ALPE has a good short-term prognosis. Renal function generally recovers within three days to several weeks [1]. Early correction of hypovolaemia with intravenous fluids accelerates recovery [3]. Haemodialysis may be required in patients with severe renal failure, but they likewise make a good recovery [1-3]. In the present case, the maximum serum creatinine level was higher in the first ALPE episode, possibly due to a delayed presentation and commencement of intravenous fluids. The renal function trended abnormally after this episode, indicating that recurrence without any symptoms might have occurred.

The long-term prognosis of ALPE remains unclear due to a lack of long-term data. Kikuchi et al. [14] demonstrated chronic renal lesions in a 38-year-old male who experienced at least four ALPE episodes [14]. ALPE recurrence occurs in up to a quarter of patients, with most cases in patients with renal hypouricaemia $[1,3]$. The present case had ALPE recurrence without hypouricaemia. He will likely continue to suffer from recurrent episodes with ongoing intense anaerobic exercise, and we predict that this will eventually cause a permanent renal deficit and result in a negative outcome. Therefore, we advised the patient to avoid intense elite-level anaerobic exercise.

Further reasoning for the patient having to discontinue elite-level sport was that the professional sports team's management could not continue his employment after considering their health and safety responsibilities. Recurrent episodes of renal injury caused by elitelevel sport could effectively be construed as a workplace injury in New Zealand. Similar employment challenges may present for other professional athletes and occupations that require intense exercise. There have been cases in Japan of work-related ALPE in a police officer and a professional cyclist, although they continued their careers despite the ongoing risk of recurrence [15]. Whilst prohibiting intense anaerobic exercise is an effective strategy to prevent ALPE recurrence, this can have significant social, economic, and psychological implications for patients.

In summary, ALPE is possibly an under-recognized condition worldwide and may not be ascribable to specific population genotypes. The diagnosis of ALPE should be considered in all patients who present with loin or abdominal pain accompanied by AKI. Long-term outcomes of recurrent ALPE remain unclear and preventative strategies should be implemented to preserve renal function. The present case highlights that patients with or without renal hypouricaemia can develop recurrent ALPE, that recurrent ALPE might occur without symptoms, and that successful prevention can be achieved by avoiding intense anaerobic exercise.

\section{Statement of Ethics}

This study was approved by the Auckland Health Research Ethics Committee. Written informed consent was obtained from the patient for publication of this case report and accompanying images.

\section{Conflict of Interest Statement}

The authors have no conflicts of interest to declare.

\section{Karger'}




\section{Funding Sources}

No funding was required for this study.

\section{Author Contributions}

Richard Kelly, Alana Harper, and David Semple were responsible for the clinical assessment and management of the patient. Richard Kelly prepared the draft manuscript. All the authors participated in editing the final manuscript.

\section{References}

1 Ishikawa I. Acute renal failure with severe loin pain and patchy renal ischemia after anaerobic exercise in patients with or without renal hypouricemia. Nephron. 2002;91(4):559-70.

2 Lee J, Lee SW, Lee JW, Chin HJ, Joo KW, Kim YS, et al. Clinical characteristics of acute renal failure with severe loin pain and patchy renal vasoconstriction. Kidney Res Clin Pract. 2012;31(3):170-6.

3 Ohta T, Sakano T, Igarashi T, Itami N, Ogawa T. Exercise-induced acute renal failure associated with renal hypouricaemia: results of a questionnaire-based survey in Japan. Nephrol Dial Transplant. 2004;19(6):144753.

4 Stiburkova B, Taylor J, Marinaki AM, Sebesta I. Acute kidney injury in two children caused by renal hypouricaemia type 2. Pediatr Nephrol. 2012;27(8):1411-5.

5 Simpson A, Dornan R, Turner A. Recurrent AKI with loin pain in a Caucasian competitive cyclist. Urate is part of the story. In: UK Kidney Week. Brighton; 2019. June 3-June 5. Abstract 428.

6 Ouellet G, Lin S, Nolin L, Bonnardeaux A. Hereditary renal hypouricemia in a Caucasian patient: a case report and review of the literature. Nephrol Ther. 2009;5(6):568-71.

7 Bahat H, Dinour D, Ganon L, Feldman L, Holtzman EJ, Goldman M. Non-urate transporter 1-related renal hypouricemia and acute renal failure in an Israeli-Arab family. Pediatr Nephrol. 2009;24(5):999-1003.

8 Bardak S, Turgutalp K, Kiykim A. Exercise-induced acute kidney injury: a case of renal hypouricemia. Turk Neph Dial Transpl. 2015;24(2):217-9.

9 Bagley WH, Yang H, Shah KH. Rhabdomyolysis. Intern Emerg Med. 2007;2(3):210-8.

10 Furumatsu Y, Nagasawa Y, Hamano T, Iwatani H, Imai E. Acute renal failure with severe loin pain after anaerobic exercise (ALPE): detection of patchy renal ischaemia by contrast-enhanced colour Doppler. NDT Plus. 2007;1(2):120-1.

11 Ishikawa I, Ishii H, Saito T, Yuri T, Shinoda A, Urashima S. Increased patchy renal accumulation of $99 \mathrm{mTc}-$ methylene diphosphonate in a patient with severe loin pain after exercise. Nephron. 1987;47(1):29-31.

12 Ohta K, Yokoyama T, Shimizu M, Mizuno K, Sakazume S, Fujiki T, et al. Diffusion-weighted MRI of exerciseinduced acute renal failure (ALPE). Pediatr Nephrol. 2011;26(8):1321-4.

13 Ames BN, Cathcart R, Schwiers E, Hochstein P. Uric acid provides an antioxidant defense in humans against oxidant- and radical-caused aging and cancer: a hypothesis. Proc Natl Acad Sci U S A. 1981;78(11):6858-62.

14 Kikuchi Y, Koga H, Yasutomo Y, Kawabata Y, Shimizu E, Naruse M, et al. Patients with renal hypouricemia with exercise-induced acute renal failure and chronic renal dysfunction. Clin Nephrol. 2000;53(6):467-72.

15 Shimizu Y, Wakabayashi K, Totsuka A, Hayashi Y, Nitta S, Hara K, et al. Exercise-induced acute kidney injury in a police officer with hereditary renal hypouricemia. Case Rep Nephrol Dial. 2019;9(2):92-101. 